Research Article

\title{
Evaluation of Growth and Development of Adolescents' Dental Arch Asymmetry with Normal Occlusion Using Three- Dimensional Digital Models
}

\author{
Dapeng Yang $\mathbb{D}^{1}$ Shiyu Ding, ${ }^{2}$ and Peipei $\mathrm{Li}^{1}$ \\ ${ }^{1}$ Department of Orthodontics, Tangshan Union Medical College Hospital, Tangshan 063000, Hebei, China \\ ${ }^{2}$ Department of Stomatology, Tangshan Voccational \& Technical College, Tangshan 063000, Hebei, China \\ Correspondence should be addressed to Dapeng Yang; young_pushu@163.com
}

Received 14 September 2020; Revised 23 April 2021; Accepted 21 May 2021; Published 4 June 2021

Academic Editor: Mihajlo Jakovljevic

Copyright (C) 2021 Dapeng Yang et al. This is an open access article distributed under the Creative Commons Attribution License, which permits unrestricted use, distribution, and reproduction in any medium, provided the original work is properly cited.

Objective. The purpose of this study was to observe the dental arch asymmetry in 12-year-olds with normal occlusion during the early permanent dentition stage. Methods. Ninety-two 12-year-old students (46 males and 46 females) who had normal occlusion during early permanent dentition were selected from a junior high school in Tangshan, China. Once per year for three consecutive years, a dental cast was obtained from each subject, and the cast was scanned with a 3D digital scanner (R700 3D). The median palatal plane (MPP) and transverse palatal plane (TPP) were used as the reference plane for the transverse and anteroposterior measurements, respectively. Results. Most of the dental arch asymmetry indicators decreased with age, but these differences were not statistically significant. The values of the midincisal edge of the upper central incisors (U1), midincisal edge of the upper lateral incisors (U2), upper canine cusp tip (U3), upper first premolar buccal cusp tip (U4), upper second premolar buccal cusp tip (U5), upper first molar mesiobuccal cusp tip (U6MB), and upper first molar distobuccal cusp tip (U6DB) to the TPP were $0.019 \mathrm{~mm}$, $0.279 \mathrm{~mm}, 0.017 \mathrm{~mm}, 0.016 \mathrm{~mm}, 0.016 \mathrm{~mm}, 0.027 \mathrm{~mm}$, and $0.200 \mathrm{~mm}$, respectively; these values were larger in males than in females $(P<0.05)$. The values of $2-5,6 \mathrm{MB}$, and 6DB-TPP were $0.154 \mathrm{~mm}, 0.102 \mathrm{~mm}, 0.119 \mathrm{~mm}, 0.259 \mathrm{~mm}, 0.206 \mathrm{~mm}$, and $0.123 \mathrm{~mm}$, respectively, larger in the mandibular than in the maxillary dental arch $(P<0.05)$. The values of the midincisal edge of the lower central incisors (L1), midincisal edge of the lower lateral incisors (L2), lower canine cusp tip (L3), lower first premolar buccal cusp tip (L4), lower second premolar buccal cusp tip (L5), lower first molar mesiobuccal cusp tip (L6MB), and lower first molar distobuccal cusp tip (L6DB) to the MPP were $0.399 \mathrm{~mm}, 0.197 \mathrm{~mm}, 0.258 \mathrm{~mm}, 0.248 \mathrm{~mm}, 0.214 \mathrm{~mm}, 0.575 \mathrm{~mm}$, and $0.531 \mathrm{~mm}$, respectively, larger than L1-5, L6MB, and L6DB-TPP $(P<0.05)$. Conclusion. The asymmetry of the dental arch in 12 -to15 -year-olds with normal occlusion did not change significantly with age. The anteroposterior asymmetry of the maxillary dental arch is larger in males than in females. With the exception of the central incisor, the anteroposterior asymmetry of the mandibular dental arch is larger than that of the maxillary dental arch. The transverse asymmetry of the mandibular dental arch is larger than the anteroposterior asymmetry.

\section{Introduction}

Maxillofacial asymmetry includes skeletal asymmetry, dental arch asymmetry, and soft tissue asymmetry [1-3]. Dental arch asymmetry has been studied extensively $[4,5]$ and is related to many diseases. Manfredini et al. [6] found that patients with temporomandibular joint disorder have complicated dental arch asymmetry. Mild facial asymmetry is correlated with dental arch asymmetry [7]. Dental arch asymmetry has been studied in patients with different degrees of malocclusion based on Angle's classifications. Skrinjaric et al. [8] found that the degree of fluctuation in dental arch asymmetry is highest in class III patients. Yu et al. [9] found that the mandibular deviation of class III dental arches can misdirect the teeth to the buccolingual direction, leading to dental arch asymmetry. Veli et al. [10] found that asymmetry in dental arches with class II subdivision malocclusion does not change with increasing age. 
Among studies of dental arch asymmetry at different ages, Kula et al. [11] found that the maxillary and mandibular dental arches of children with deep overjet are asymmetric and that the lateral and anteroposterior asymmetric dental arches are located at the position of the maxillary first permanent molars. Maurice and Kula [12] found that sagittal asymmetry in the dental arches during the mixed dentition period is more serious than anteroposterior asymmetry. Ferrario et al. [13] found slight asymmetry in the dental arches of adults and concluded that such asymmetry is normal and acceptable.

No longitudinal study has examined the asymmetry of dental arches with normal occlusion at the beginning of permanent dentition. The purpose of this study was to follow up and observe adolescents aged 12 years with normal occlusion for two consecutive years. Three-dimensional digital models were used to observe the variations in dental arch asymmetry with respect to the subjects' age and sex; the differences in maxillary and mandibular dental arch asymmetry and sagittal and anteroposterior dental arch asymmetry were studied.

\section{Materials and Methods}

In this study, 12-year-old students who had normal occlusion during the early permanent dentition stages were selected from a junior high school in Tangshan, China. The selection criteria were as follows: (1) subjects with class I molar relationship; (2) subjects with symmetrical facial contours and without obvious deformity, prominent or retracted contour of the face; (3) subjects without deformity or missing teeth; (4) subjects without history of orthodontic treatment; (5) subjects without crossbite; and (6) subjects without a history of facial trauma or changes in physical condition.

In total, 92 research subjects were included (46 males and 46 females). All dental casts were scanned by a 3D laser scanner (R7003D Dental Scanner; 3Shape A/S, Copenhagen, Denmark). The models were analyzed by a researcher using 3Shape OrthoAnalyzer analysis software (version 10.7.10).

The definitions and measurements of the mark points of these linear measurements are shown in Table 1. The reference plane for the median palatal plane (MPP) was drawn through two landmarks on the median palatal raphe. One landmark was identified as the point on the median palatal raphe adjacent to the second rugae, and the second landmark was approximately one centimeter distal to the first point on the median palatal raphe. The reference plane for the transverse palatal plane (TPP) was drawn vertical to the corresponding MPP through a common landmark (common point at the intersection of the maxillary and the mandibular dental arches (MPPB)) at the distal edges. The linear measurements of dental arch asymmetry included transverse measurements and anteroposterior measurements. Transverse measurements were taken $90^{\circ}$ from the MPP to the bilateral landmarks. Similarly, anteroposterior measurements were taken $90^{\circ}$ from the TPP to the bilateral landmarks. The measurements are shown in Figure 1.
The difference in distance to the MPP or TPP between the two sides was analyzed to evaluate dental arch asymmetry. To do so, the left side value was subtracted from the right side value. A positive or negative value indicates that the right dental arch is larger or smaller than the left dental arch, respectively. The mean absolute difference was calculated as the difference between two groups of bilateral MPP or TPP markers in all subjects. The value of the absolute difference indicating true severity of asymmetry was set at $2.0 \mathrm{~mm}$.

Twenty cases were randomly selected and remeasured by the same examiner (Dapeng Yang) to calculate the method error. The variability of the examiner's repeated measurements was assessed by calculating the interclass correlation coefficient and measurement error for each linear distance. The mean difference of each variable between consecutive traits was compared with the paired t test. There was no significant difference between the two measurements.

\section{Results}

The means and standard deviations of dental arch asymmetry studied for three consecutive years in 12-year-old adolescents with normal occlusion are summarized in $\mathrm{Ta}-$ bles 2 and 3. The changes in dental arch asymmetry for three consecutive years are shown in Figure 2. The figure shows that the differences in distances from the MPP and TPP between teeth with the same name on both sides of the maxilla and mandible were less than $2 \mathrm{~mm}$, indicating that there is no absolute asymmetry in the maxillary and mandibular dental arches. In addition, most of the differences decreased over three years, but not significantly. A comparison of dental arch asymmetry between males and females is shown in Figure 3. There were no differences between males and females in the values of U1-5, U6MB, and U6DB-MPP (Figure 3(a)); the values of L1-5, U6MB, and U6DB-MPP (Figure 3(c)); the values of L1-5, U6MB, and U6DB-TPP (Figure 3(d)). However, the values of U1-5, $\mathrm{U} 6 \mathrm{MB}$, and U6DB-TPP were $0.019 \mathrm{~mm}, 0.279 \mathrm{~mm}$, $0.017 \mathrm{~mm}, 0.016 \mathrm{~mm}, 0.016 \mathrm{~mm}, 0.027 \mathrm{~mm}$, and $0.200 \mathrm{~mm}$, respectively, larger in males than in females $(P<0.05)$ (Table 4 and Figure 3(b)). A comparison of asymmetry between the maxillary and mandibular dental arches is shown in Figure 4. There were no differences in the values of $1-5,6 \mathrm{MB}$, and 6DB-MPP between the maxillary and mandibular dental arches (Figure 4(a)).However, the values of $2-5,6 \mathrm{MB}$, and $6 \mathrm{DB}-\mathrm{TPP}$ were $0.154 \mathrm{~mm}, 0.102 \mathrm{~mm}$, $0.119 \mathrm{~mm}, 0.259 \mathrm{~mm}, 0.206 \mathrm{~mm}$, and $0.123 \mathrm{~mm}$, respectively, larger in the mandibular dental arch than in the maxillary dental arch $(P<0.05)$ (Table 5 and Figure $4(\mathrm{~b}))$. Figure 5 illustrates the lateral and anteroposterior asymmetry of the maxillary and mandibular dental arches. There were no differences in the values of U1-5, U6MB, U6DB-MPP, U1-5, $6 \mathrm{MB}$, and 6DB-TPP (Figure 5(a)). However, the values of L1-5, L6MB, and L6DB-MPP were $0.399 \mathrm{~mm}, 0.197 \mathrm{~mm}$, $0.258 \mathrm{~mm}, 0.248 \mathrm{~mm}, 0.214 \mathrm{~mm}, 0.575 \mathrm{~mm}$, and $0.531 \mathrm{~mm}$, respectively, larger than L1-5, L6MB, and L6DB-TPP $(P<0.05)$ (Table 6 and Figure 5(b)). 
Table 1: Definitions of acronyms for cast landmarks.

\begin{tabular}{lc}
\hline Acronym & Description \\
\hline U1/L1 & Midincisal edge of upper/lower central incisors \\
U2/L2 & Midincisal edge of upper/lower lateral incisors \\
U3/L3 & Upper/lower canine cusp tip \\
U4/L4 & Upper/lower first premolar buccal cusp tip \\
U5/L5 & Upper/lower second premolar buccal cusp tip \\
U6MB/L6MB & Upper/lower first molar mesiobuccal cusp tip \\
U6DB/L6DB & Upper/lower first molar distobuccal cusp tip \\
MPP & Median palatal plane \\
TPP & Transpalatal plane \\
MPPB & Common point at the intersection of the maxillary MPP and the mandibular MPP
\end{tabular}

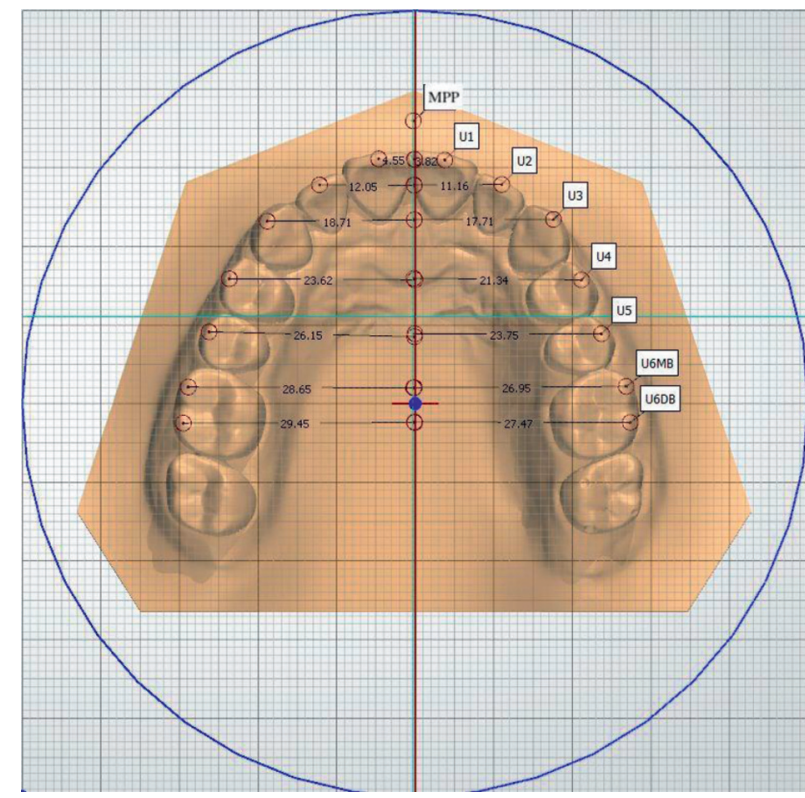

(a)

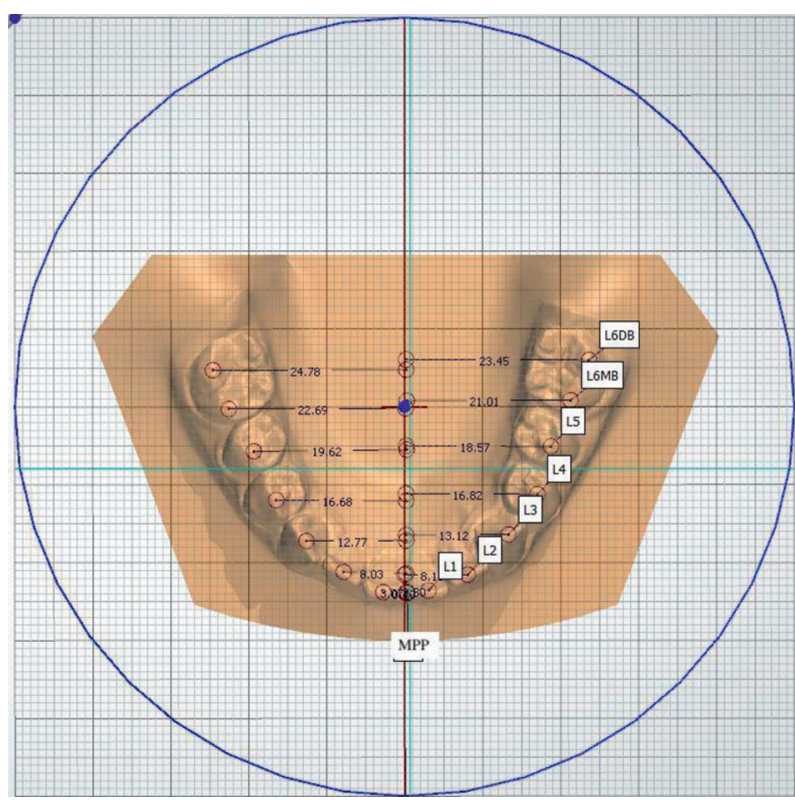

(c)

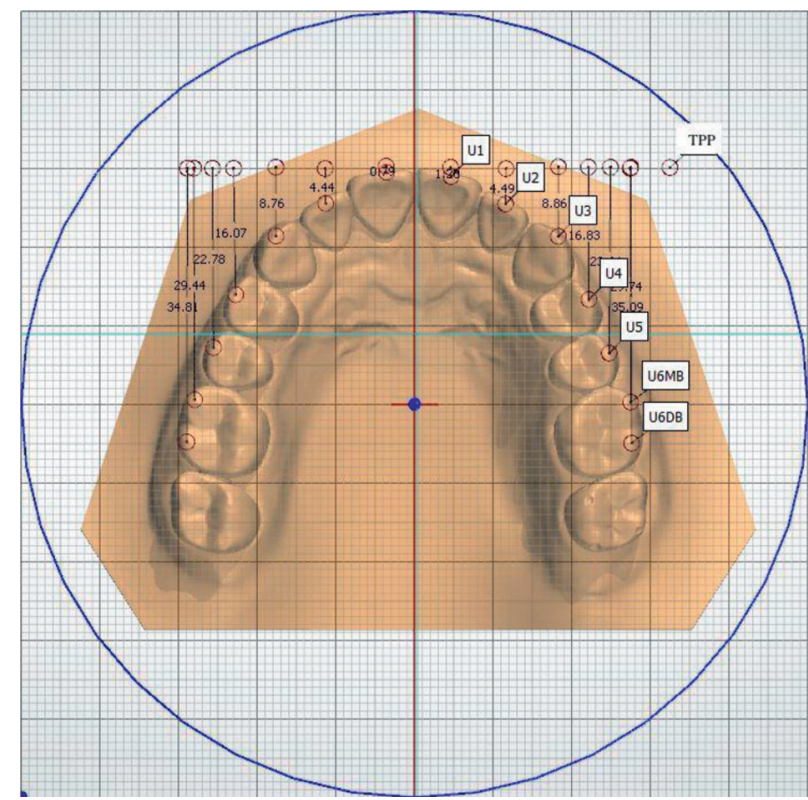

(b)

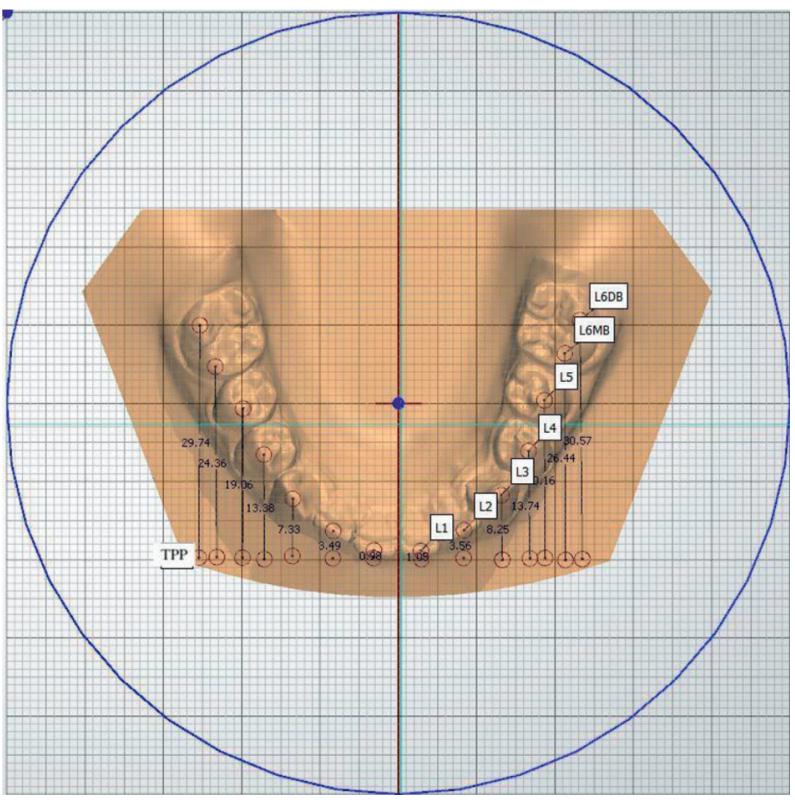

(d)

Figure 1: (a) Linear measurements from bilateral tooth landmarks to the MPP on the maxillary dental arch. (b) Linear measurements from bilateral tooth landmarks to the TPP on the maxillary dental arch. (c) Linear measurements from bilateral tooth landmarks to the MPP on the mandibular dental arch. (d) Linear measurements from bilateral tooth landmarks to the TPP on the mandibular dental arch. 
TABLE 2: Statistical comparisons of absolute differences between horizontal and vertical measurements of maxillary bilateral dental landmarks using ordinary one-way ANOVA.

\begin{tabular}{|c|c|c|c|c|c|c|c|c|c|}
\hline \multirow{2}{*}{ Measurement (mm) } & \multicolumn{2}{|c|}{12 years $(n=92)$} & \multicolumn{2}{|c|}{13 years $(n=92)$} & \multicolumn{2}{|c|}{14 years $(n=92)$} & \multicolumn{2}{|c|}{15 years $(n=92)$} & \multirow{2}{*}{$P$} \\
\hline & Mean & $\mathrm{SD}$ & Mean & SD & Mean & $\mathrm{SD}$ & Mean & $\mathrm{SD}$ & \\
\hline U1 to MPP & -0.465 & 2.469 & -0.252 & 0.371 & 0.725 & 0.452 & 0.725 & 0.652 & 0.548 \\
\hline $\mathrm{U} 2$ to $\mathrm{MPP}$ & -0.178 & 0.537 & 0.254 & 0.399 & 0.536 & 0.744 & 0.236 & 0.544 & 0.377 \\
\hline U3 to MPP & -0.246 & 0.386 & -0.226 & 0.626 & -0.148 & 0.774 & -0.248 & 0.854 & 0.625 \\
\hline $\mathrm{U} 4$ to $\mathrm{MPP}$ & 0.120 & 0.865 & 0.133 & 0.703 & -0.547 & 1.239 & -0.477 & 1.109 & 0.414 \\
\hline U5 to MPP & -0.282 & 0.298 & -0.330 & 0.833 & 0.146 & 0.672 & 0.216 & 0.602 & 0.262 \\
\hline U6MB to MPP & -0.237 & 1.166 & -0.317 & 0.576 & -0.160 & 0.836 & -0.260 & 0.632 & 0.906 \\
\hline U6DB to MPP & 0.164 & 0.634 & 0.352 & 1.165 & 0.498 & 0.864 & 0.498 & 0.664 & 0.527 \\
\hline $\mathrm{U} 1$ to TPP & 0.015 & 0.115 & -0.129 & 0.475 & 0.068 & 0.155 & 0.048 & 0.175 & 0.318 \\
\hline $\mathrm{U} 2$ to $\mathrm{TPP}$ & -0.145 & 0.324 & -0.144 & 0.523 & -0.228 & 0.312 & -0.228 & 0.841 & 0.526 \\
\hline U3 to TPP & -0.239 & 0.534 & -0.329 & 0.622 & 0.322 & 0.401 & 0.222 & 0.465 & 0.319 \\
\hline $\mathrm{U} 4$ to TPP & -0.150 & 0.633 & -0.594 & 0.670 & -0.598 & 0.694 & -0.398 & 0.514 & 0.302 \\
\hline U5 to TPP & -0.365 & 1.124 & -0.598 & 0.213 & -0.379 & 0.595 & -0.579 & 0.575 & 0.256 \\
\hline U6MB to TPP & -0.363 & 1.183 & -0.764 & 0.374 & -0.855 & 0.382 & -0.655 & 0.612 & 0.252 \\
\hline U6DB to TPP & -0.423 & 1.378 & -0.679 & 0.648 & -0.342 & 0.732 & -0.442 & 0.732 & 0.446 \\
\hline
\end{tabular}

NS indicates nonsignificant; ${ }^{*} P=0.05 ;{ }^{* *} P=0.01 ;{ }^{* * *} P=0.001$.

TABLE 3: Statistical comparisons of absolute differences between horizontal and vertical measurements of mandibular bilateral dental using ordinary one-way ANOVA.

\begin{tabular}{|c|c|c|c|c|c|c|c|c|c|}
\hline \multirow{2}{*}{ Measurement (mm) } & \multicolumn{2}{|c|}{12 years $(n=92)$} & \multicolumn{2}{|c|}{13 years $(n=92)$} & \multicolumn{2}{|c|}{14 years $(n=92)$} & \multicolumn{2}{|c|}{15 years $(n=92)$} & \multirow{2}{*}{$P$} \\
\hline & Mean & $\mathrm{SD}$ & Mean & $\mathrm{SD}$ & Mean & SD & Mean & SD & \\
\hline L1 to MPP & -0.169 & 0.338 & 0.218 & 0.531 & -0.111 & 0.344 & -0.211 & 0.546 & 0.539 \\
\hline L2 to MPP & -0.452 & 0.598 & 0.199 & 0.642 & -0.223 & 0.534 & -0.243 & 0.544 & 0.376 \\
\hline L3 to MPP & -0.193 & 0.775 & 0.365 & 1.434 & -0.142 & 1.147 & -0.143 & 1.147 & 0.492 \\
\hline L4 to MPP & -0.288 & 0.716 & 0.377 & 1.152 & 0.260 & 0.952 & 0.161 & 0.872 & 0.121 \\
\hline L5 to MPP & -0.117 & 1.279 & 0.335 & 1.431 & 0.356 & 0.665 & 0.353 & 0.615 & 0.723 \\
\hline L6MB to MPP & 0.314 & 1.352 & 0.478 & 1.517 & 0.229 & 0.614 & 0.237 & 0.714 & 0.863 \\
\hline L6DB to MPP & -0.182 & 0.553 & 0.403 & 1.339 & 0.627 & 0.551 & 0.617 & 0.451 & 0.544 \\
\hline L1 to TPP & 0.115 & 0.527 & 0.176 & 0.131 & 0.129 & 0.262 & 0.129 & 0.142 & 0.784 \\
\hline $\mathrm{L} 2$ to $\mathrm{TPP}$ & 0.192 & 0.445 & -0.071 & 0.341 & -0.373 & 0.347 & -0.343 & 0.347 & 0.686 \\
\hline L3 to TPP & -0.122 & 0.748 & -0.388 & 0.427 & -0.323 & 0.543 & -0.423 & 0.542 & 0.201 \\
\hline $\mathrm{L} 4$ to $\mathrm{TPP}$ & -0.168 & 1.539 & -0.173 & 0.482 & -0.551 & 0.776 & -0.651 & 0.956 & 0.474 \\
\hline L5 to TPP & -0.632 & 1.197 & -0.498 & 1.246 & -0.648 & 0.875 & -0.747 & 0.795 & 0.473 \\
\hline L6MB to TPP & 0.273 & 1.436 & -0.363 & 0.796 & -0.755 & 0.897 & -0.753 & 0.875 & 0.181 \\
\hline L6DB to TPP & -0.121 & 1.571 & -0.213 & 1.384 & -0.543 & 0.514 & -0.513 & 0.954 & 0.332 \\
\hline
\end{tabular}

NS indicates nonsignificant; ${ }^{*} P=0.05 ;{ }^{* *} P=0.01 ;{ }^{* * *} P=0.001$.

\section{Discussion}

The study by Maurice [12] of the asymmetry of normal occlusion in the mixed dentition period found that dental arches are not absolutely symmetrical, but a difference of no more than $2 \mathrm{~mm}$ in the distances from the bilateral corresponding teeth to the reference line is considered clinically acceptable. In Figure 2(a), all values, except U2-MPP, are negative, indicating that the maxillary left dental arch is larger than the right dental arch, which is consistent with the findings of Slaj et al. [14]. However, Slaj et al. reported that dental arch symmetry in adolescents with normal occlusion deteriorated during the mixed dentition period. By contrast, Figure 2 shows that the values of most measurement indices decreased, indicating that the dental arch is symmetric; however, the decrease was not statistically significant. According to our analysis of the results, this decrease occurred because the average starting age of the subjects in this study was 12 years, which marks the beginning of permanent dentition. Dental arches tend to be stable with increasing age, and thus, the study findings are consistent with the cited literature.

Previous reports on differences in the sizes of the dental arches between sexes showed that the length, width, and height of dental arches are all larger in males than in females [15]. In terms of dental arch asymmetry, most articles in the literature conclude that there is no difference between male and female subjects [16]. However, in this study, we found that the anteroposterior asymmetry of the maxillary arches was significantly larger in males than in females. This finding is consistent with the findings of Skrinjaric et al. [8] and may be attributed to the fact that females reach peak growth and development earlier than males; therefore, their dental arches tend to stabilize earlier than males. Since the peak growth and development of women are earlier than those of men, their dental arches can be stabilized earlier, and the age 


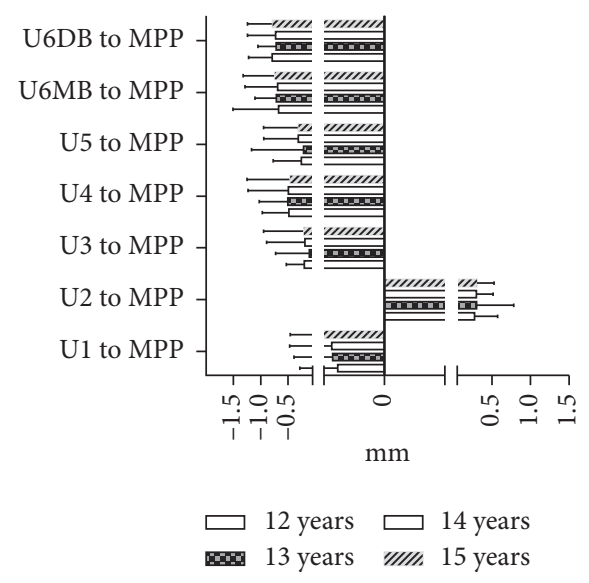

(a)

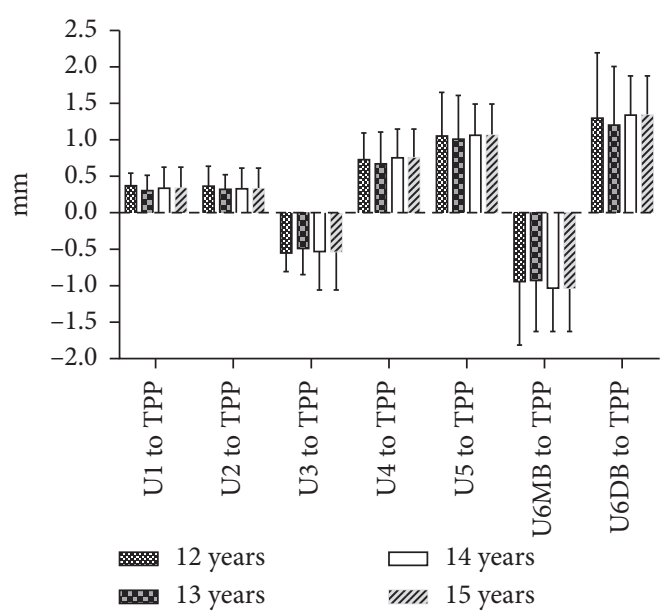

(b)

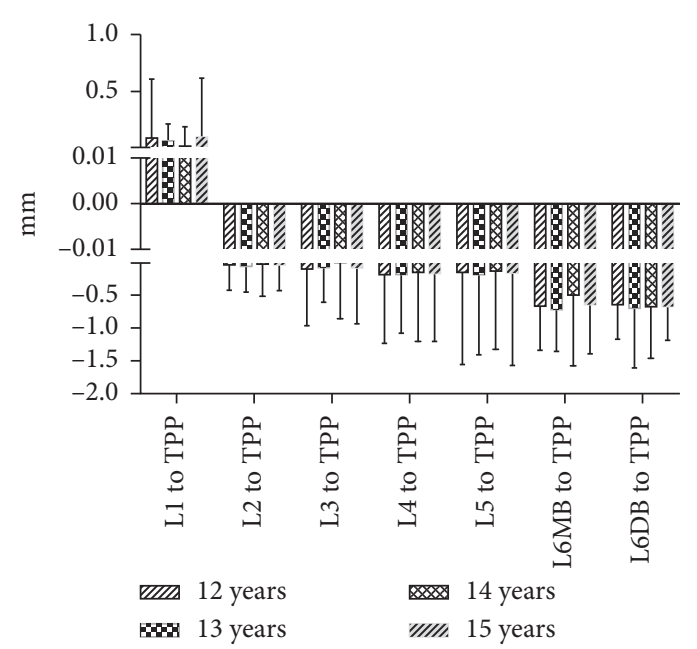

(d)

Figure 2: (a) Changes in the lateral asymmetry of the maxillary dental arch for two consecutive years. (b) Changes in the anteroposterior asymmetry of the maxillary dental arch for two consecutive years. (c) Changes in the lateral asymmetry of the mandibular dental arch for two consecutive years. (d) Changes in the anteroposterior asymmetry of the mandibular dental arch for two consecutive years.

of orthodontic surgery can be advanced to 16 years. The disadvantage is that women who need early orthodontic treatment should be detected and treated early by an orthodontist to avoid missing the best timing for early intervention.

De Araujo et al. $[17,18]$ analyzed asymmetry in dental arches with normal occlusion as belonging to Angle's class II and class I. The results showed that the asymmetry in the mandibular dental arch was larger than that in the maxillary dental arch. Nie and Jin [19] compared the asymmetry of dental arches in patients with different occlusions based on Angle's classification and found that asymmetry is larger in the mandibular dental arch than in the maxillary dental arch, except for class III occlusion cases. As shown in Figure 4, the lateral and anteroposterior asymmetry are larger in the mandibular dental arch than in the maxillary dental arch except for the position of the central incisors. In addition, the anteroposterior asymmetry is significantly larger in the mandibular dental arch than in the maxillary dental arch
(Figure 4(b)). This finding is consistent with the conclusions of the cited literature.

The asymmetry of the mandibular dental arch is larger than that of the maxillary arch, which is related to not only the asymmetry of the dental arch but also the asymmetry of the jawbone. Kusayama et al. [20] investigated the relationship between lateral abnormalities of the dental arch and jawbone asymmetry and found a high correlation between dental arch abnormalities and skeletal asymmetry. Mandibular movement can increase the incidence of mandibular asymmetry but can also compensate for the asymmetry of the dental arch.

Maurice and Kula [12] found that the lateral asymmetry in dental arches is larger than the anteroposterior asymmetry. In this study, the lateral and anteroposterior asymmetries of the maxillary and mandibular dental arches were compared in detail. As shown in Figure 5(a), the differences between the distances of the corresponding teeth on both sides of the maxillary to the MPP plane were larger than the 


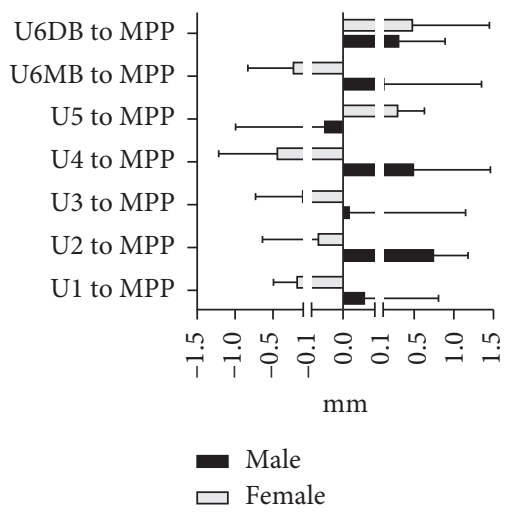

(a)

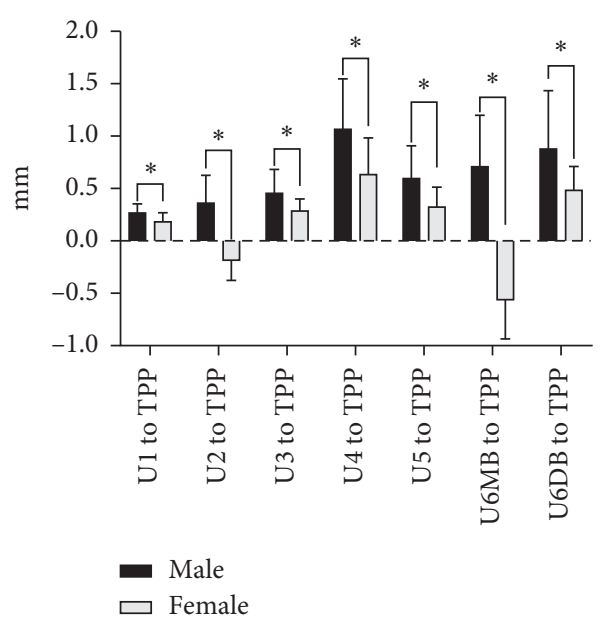

(b)

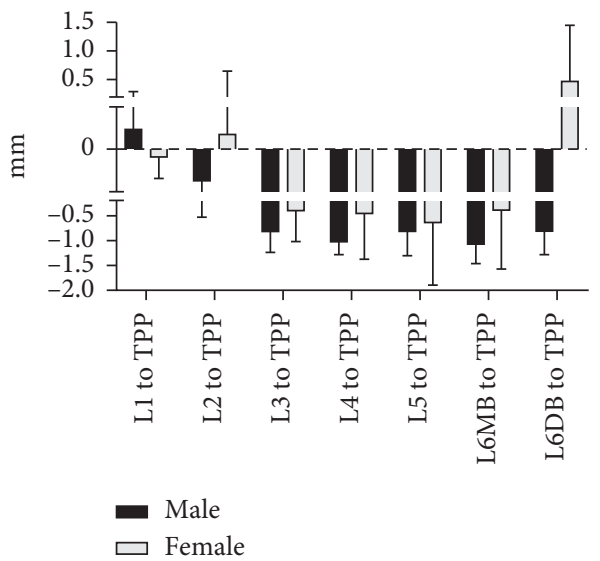

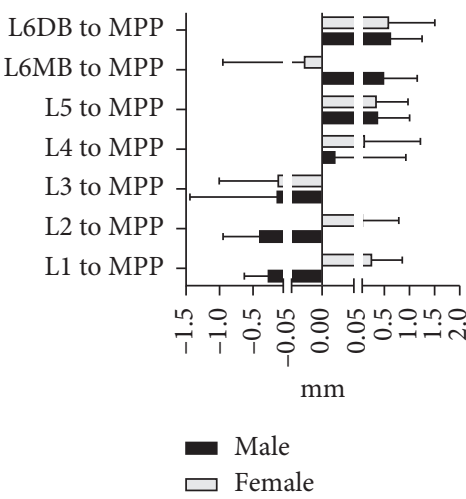

(c)

(d)

FiguRE 3: (a) Comparison of the asymmetries in the maxillary lateral arch between male and female. (b) Comparison of the asymmetries in the maxillary anteroposterior arch between male and female. (c) Comparison of the asymmetries in the mandibular lateral arch between male and female. (d) Comparison of the asymmetries in the mandibular anteroposterior arch between male and female. NS indicates nonsignificant; ${ }^{*} P=0.05 ;{ }^{* *} P=0.01 ;{ }^{* *} P=0.001$.

TABLE 4: Statistical comparisons of absolute differences between male and female measurements of U1-5, U6MB, and U6DB-TPP using paired t-test analysis.

\begin{tabular}{|c|c|c|c|c|c|}
\hline \multirow{2}{*}{ Measurement $(\mathrm{mm})$} & \multicolumn{2}{|c|}{ Male $(n=92)$} & \multicolumn{2}{|c|}{ Female $(n=92)$} & \multirow{2}{*}{$P$} \\
\hline & Mean & $\mathrm{SD}$ & Mean & $\mathrm{SD}$ & \\
\hline U1 to TPP & 0.212 & 0.334 & 0.193 & 0.203 & $*$ \\
\hline $\mathrm{U} 2$ to $\mathrm{TPP}$ & 0.393 & 0.553 & 0.114 & 0.244 & $*$ \\
\hline $\mathrm{U} 3$ to TPP & 0.312 & 0.293 & 0.295 & 0.267 & $*$ \\
\hline $\mathrm{U} 4$ to TPP & 0.652 & 0.753 & 0.636 & 0.926 & $*$ \\
\hline U5 to TPP & 0.564 & 0.675 & 0.537 & 0.774 & * \\
\hline U6MB to TPP & 0.593 & 1.125 & 0.563 & 0.822 & $*$ \\
\hline U6DB to TPP & 0.772 & 1.633 & 0.572 & 0.641 & $*$ \\
\hline
\end{tabular}

NS indicates nonsignificant; ${ }^{*} P=0.05 ;{ }^{* *} P=0.01 ;{ }^{* * *} P=0.001$.

distances of the corresponding teeth on both sides of the maxillary to the TPP plane, indicating that the lateral asymmetry of the maxillary dental arch is larger than the anteroposterior asymmetry, but this difference was not statistically significant. Figure 5(b) shows that the lateral asymmetry of the mandibular dental arch is significantly larger than the anteroposterior asymmetry. This finding is related to the selected sample. The subjects in this study were 


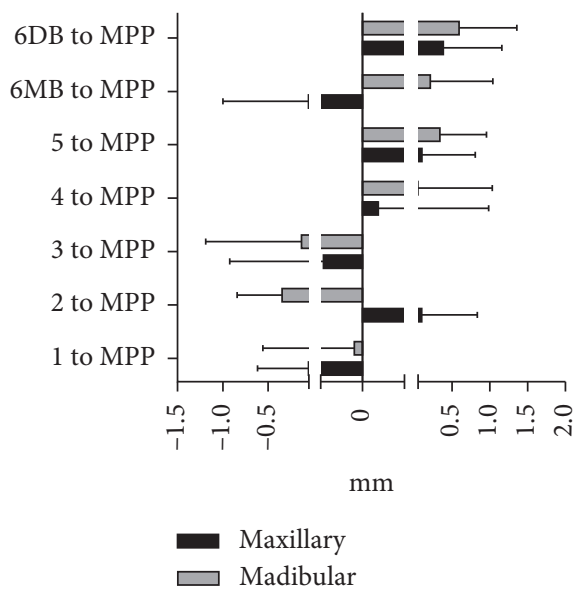

(a)

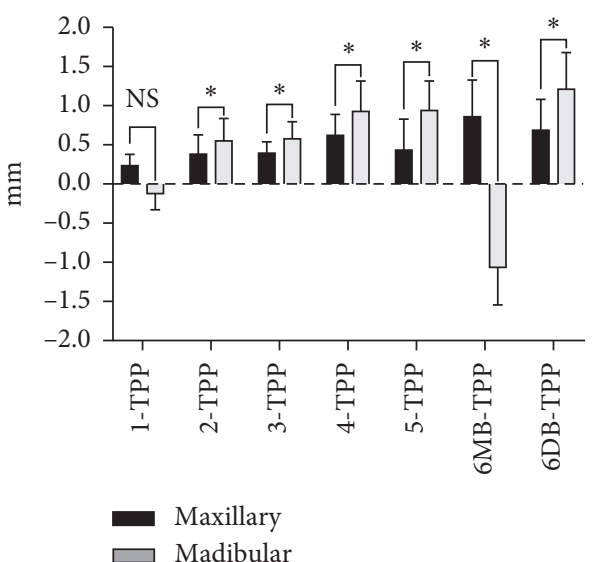

(b)

Figure 4: (a) Comparison of the lateral asymmetries in the maxillary and mandibular dental arches. (b) Comparison of the anteroposterior asymmetries in the maxillary and mandibular dental arches. NS indicates nonsignificant; ${ }^{*} P=0.05 ;{ }^{* *} P=0.01 ;{ }^{* * *} P=0.001$.

TABLE 5: Statistical comparisons of absolute differences between maxillary and mandibular measurements of 1-5, 6MB, 6DB-TPP using paired t-test analysis.

\begin{tabular}{|c|c|c|c|c|c|}
\hline \multirow{2}{*}{ Measurement (mm) } & \multicolumn{2}{|c|}{ Maxillary $(n=92)$} & \multicolumn{2}{|c|}{ Mandibular $(n=92)$} & \multirow{2}{*}{$P$} \\
\hline & Mean & SD & Mean & SD & \\
\hline 1-TPP & 0.253 & 0.34 & 0.141 & 0.653 & NS \\
\hline 2-TPP & 0.248 & 0.41 & 0.402 & 0.385 & $*$ \\
\hline 3-ТPP & 0.423 & 0.42 & 0.534 & 0.519 & $*$ \\
\hline 4-ТPP & 0.647 & 0.74 & 0.766 & 1.007 & $*$ \\
\hline 5-TPP & 0.454 & 1.044 & 0.713 & 0.855 & $*$ \\
\hline 6MB-TPP & 0.882 & 1.456 & 1.088 & 1.441 & $*$ \\
\hline 6DB-TPP & 0.713 & 1.057 & 0.836 & 1.463 & * \\
\hline
\end{tabular}

NS indicates nonsignificant; ${ }^{*} P=0.05 ;{ }^{* *} P=0.01 ;{ }^{* * *} P=0.001$.

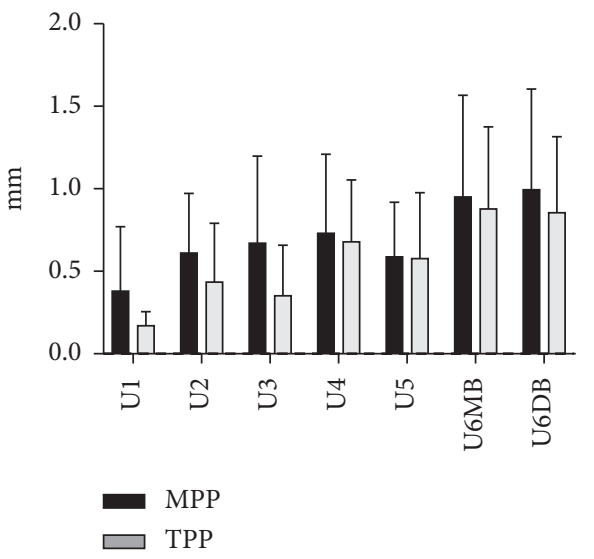

(a)

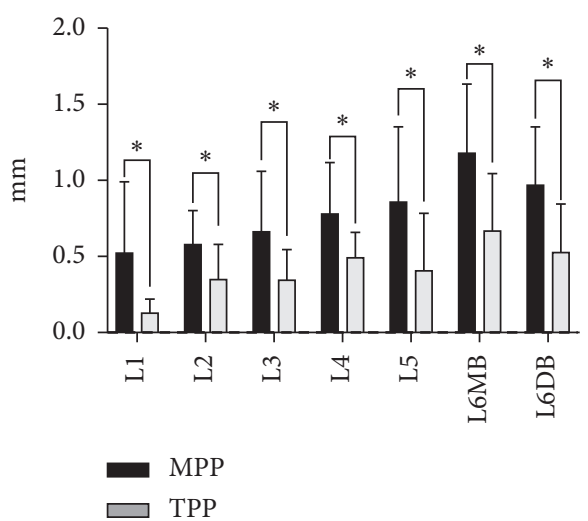

(b)

Figure 5: (a) Comparison of the lateral and anteroposterior asymmetries in the maxillary 257 dental arch. (b) Comparison of the lateral and anteroposterior asymmetries in the mandibular dental arch. NS indicates nonsignificant; ${ }^{*} P=0.05 ;{ }^{* *} P=0.01 ;{ }^{* * *} P=0.001$.

teenagers with normal occlusion and Angle's class I molar relationship. Subjects with a class II or class III relationship with anteroposterior asymmetry of the dental arch were excluded. To confirm these findings, in future studies, we can select different subjects with different Angle's classifications for sagittal and anterior-posterior comparison. 
TABLE 6: Statistical comparisons of absolute differences between measurements of L1-5, L6MB, and L6DB to TPP and MPP using paired t-test analysis.

\begin{tabular}{lccccc}
\hline \multirow{2}{*}{ Measurement $(\mathrm{mm})$} & \multicolumn{2}{c}{ MPP $(n=92)$} & \multicolumn{2}{c}{ TPP $(n=92)$} & \multirow{2}{*}{ Mean } \\
& SD & Mean & SD & \\
\hline L1 & 0.532 & 1.714 & 0.133 & 0.266 & $*$ \\
L2 & 0.650 & 0.513 & 0.453 & 0.717 & $*$ \\
L3 & 0.673 & 1.054 & 0.415 & 0.666 & $*$ \\
L4 & 0.783 & 1.116 & 0.535 & 0.427 & $*$ \\
L5 & 0.862 & 1.317 & 0.648 & 1.058 & $*$ \\
L6MB & 1.251 & 0.898 & 0.676 & 1.199 & $*$ \\
L6DB & 1.066 & 0.809 & 0.535 & 0.861 & $*$ \\
\hline
\end{tabular}

NS indicates nonsignificant; ${ }^{*} P=0.05 ;{ }^{* *} P=0.01 ;{ }^{* * *} P=0.001$.

\section{Conclusions}

(1) The asymmetry of the dental arch in 12-to-15-yearolds with normal occlusion did not change significantly with age.

(2) The anteroposterior asymmetry of the maxillary dental arch was larger in males than in females.

(3) With the exception of the central incisor, the anteroposterior asymmetry of the mandibular dental arch was larger than that of the maxillary dental arch.

(4) The transverse asymmetry of the mandibular dental arch is significantly larger than the anteroposterior asymmetry.

\section{Data Availability}

The data used to support the findings of this study are included within the article.

\section{Conflicts of Interest}

The authors declare that there are no conflicts of interest regarding the publication of this paper.

\section{Authors' Contributions}

Dapeng Yang and Shiyu Ding contributed equally to this work. Dapeng Yang is the corresponding author.

\section{Acknowledgments}

Thanks are due to Professor Bai Yuxing and Associate Professor Zhang Ke for their support in this research.

\section{References}

[1] Y.-J. Chen, C.-C. Yao, Z.-C. Chang, H.-H. Lai, K.-J. Yeh, and S.-H. Kok, "Characterization of facial asymmetry in skeletal class III malocclusion and its implications for treatment," International Journal of Oral and Maxillofacial Surgery, vol. 48, no. 12, pp. 1533-1541, 2019.

[2] R. B. Nur, D. G. Çakan, and T. Arun, "Evaluation of facial hard and soft tissue asymmetry using cone-beam computed tomography," American Journal of Orthodontics and Dentofacial Orthopedics, vol. 149, no. 2, pp. 225-237, 2016.
[3] R. Kai, D. Umeki, T. Sekiya, and Y. Nakamura, "Defining the location of the dental midline is critical for oral esthetics in camouflage orthodontic treatment of facial asymmetry," American Journal of Orthodontics and Dentofacial Orthopedics, vol. 150, no. 6, pp. 1028-1038, 2016.

[4] S. E. Bishara, P. S. Burkey, and J. G. Kharouf, "Dental and facial asymmetries: a review," The Angle Orthodontist, vol. 64, no. 2, pp. 89-98, 1994.

[5] J. W. Beyer and S. J. Lindauer, "Evaluation of dental midline position," Seminars in Orthodontics, vol. 4, no. 3, pp. 146-152, 1998.

[6] D. Manfredini, L. Lombardo, and G. Siciliani, "Dental Angle class asymmetry and temporomandibular disorders," Journal of Orofacial Orthopedics/Fortschritte der Kieferorthopädie, vol. 78, no. 3, pp. 253-258, 2017.

[7] D. Y. Shen, L. H. Hu, S. H. Zuo, Q. C. Kang, and X. L. Yan, "Evaluation of dental and craniofacial characteristics in patients with mild skeletal facial asymmetry," Shanghai Kou Qiang Yi Xue, vol. 27, no. 4, pp. 376-381, 2018.

[8] A. Skrinjaric, M. Slaj, and M. Slaj, "Fluctuating dental arch asymmetry in different malocclusion groups," Acta Stomatologica Croatica, vol. 52, no. 2, pp. 105-113, 2018.

[9] J. Yu, Y. Hu, M. Huang, J. Chen, X. Ding, and L. Zheng, “A three-dimensional analysis of skeletal and dental characteristics in skeletal class III patients with facial asymmetry," Journal of X-Ray Science and Technology, vol. 26, no. 3, pp. 449-462, 2018.

[10] I. Veli, B. Yuksel, and T. Uysal, "Longitudinal evaluation of dental arch asymmetry in Class II subdivision malocclusion with 3-dimensional digital models," American Journal of Orthodontics and Dentofacial Orthopedics, vol. 145, no. 6, pp. 763-770, 2014.

[11] K. Kula, A. Esmailnejad, and A. Hass, "Dental arch asymmetry in children with large overjets," The Angle Orthodontist, vol. 68, no. 1, pp. 45-52, 1998.

[12] T. J. Maurice and K. Kula, "Dental arch asymmetry in the mixed dentition," The Angle Orthodontist, vol. 68, no. 1, pp. 37-44, 1998.

[13] V. F. Ferrario, C. Sforza, A. Colombo, A. Miani, and A. D'Addona, "Position and asymmetry of teeth in untreated dental arches," The International Journal of Adult Orthodontics and Orthognathic Surgery, vol. 8, no. 4, pp. 277-285, 1993.

[14] M. Slaj, M. A. Jezina, T. Lauc, S. Rajić-Mestrović, and M. Miksić, "Longitudinal dental arch changes in the mixed dentition," The Angle Orthodontist, vol. 73, no. 5, pp. 509-514, 2003.

[15] H. H. Tsai and C. T. Tan, "Morphology of the palatal vault of primary dentition in transverse view," The Angle Orthodontist, vol. 74, no. 6, pp. 774-779, 2004.

[16] K. Khalaf, C. Elcock, R. N. Smith, and A. H. Brook, "Fluctuating dental asymmetry of multiple crown variables measured by an image analysis system," Archives of Oral Biology, vol. 50, no. 2, pp. 249-253, 2005.

[17] T. M. de Araujo, R. S. Wilhelm, and M. A. Almeida, "Skeletal and dental arch asymmetries in individuals with normal dental occlusions," The International Journal of Adult Orthodontics \& Orthognathic Surgery, vol. 9, no. 2, pp. 111-118, 1994.

[18] T. M. de Araujo, R. S. Wilhelm, and M. A. Almeida, "Skeletal and dental arch asymmetries in Class II division 1 subdivision malocclusions," Journal of Clinical Pediatric Dentistry, Springer, vol. 18, no. 3, pp. 181-185, 1994. 
[19] Q. Nie and J. Lin, "Analysis and comparison of dental arch symmetry between different Angle's malocclusion categories and normal occlusion," Zhonghua Kou Qiang Yi Xue Za Zhi, vol. 35 , no. 2, pp. 105-107, 2000.

[20] M. Kusayama, N. Motohashi, and T. Kuroda, "Relationship between transverse dental anomalies and skeletal asymmetry," American Journal of Orthodontics and Dentofacial Orthopedics, vol. 123, no. 3, pp. 329-337, 2003. 